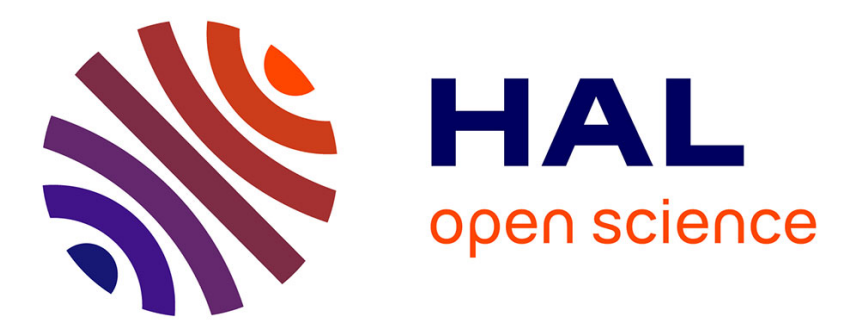

\title{
Methyl Internal Rotation in the Microwave Spectrum of o-Methyl Anisole
}

Lynn Ferres, Halima Mouhib, Wolfgang Stahl, Ha Vinh Lam Nguyen

\section{To cite this version:}

Lynn Ferres, Halima Mouhib, Wolfgang Stahl, Ha Vinh Lam Nguyen. Methyl Internal Rotation in the Microwave Spectrum of o-Methyl Anisole. ChemPhysChem, 2017, 18 (14), pp.1855-1859. 10.1002/cphc.201700276 . hal-03183103

\section{HAL Id: hal-03183103 https://hal.science/hal-03183103}

Submitted on 26 Mar 2021

HAL is a multi-disciplinary open access archive for the deposit and dissemination of scientific research documents, whether they are published or not. The documents may come from teaching and research institutions in France or abroad, or from public or private research centers.
L'archive ouverte pluridisciplinaire HAL, est destinée au dépôt et à la diffusion de documents scientifiques de niveau recherche, publiés ou non, émanant des établissements d'enseignement et de recherche français ou étrangers, des laboratoires publics ou privés. 


\title{
Methyl Internal Rotation in the Microwave Spectrum of o-Methyl Anisole
}

\author{
Lynn Ferres, ${ }^{[b]}$ Halima Mouhib, ${ }^{[b, c]}$ Wolfgang Stahl, ${ }^{[b]}$ and Ha Vinh Lam Nguyen ${ }^{*[a]}$
}

\begin{abstract}
The microwave spectrum of o-methyl anisole (2methoxytoluene), $\mathrm{CH}_{3} \mathrm{OC}_{6} \mathrm{H}_{4} \mathrm{CH}_{3}$, has been measured using a pulsed molecular jet Fourier transform microwave spectrometer operating in the frequency range $2-26.5 \mathrm{GHz}$. Conformational analysis using quantum chemical calculations at the MP2/6-311++G(d,p) level of theory yields only one stable conformer with a $C_{s}$ structure, which was assigned in the experimental spectrum. A-E splittings due to the internal rotation of the ring methyl group could be resolved and the barrier to internal rotation was determined to be $444.05(41) \mathrm{cm}^{-1}$. The experimentally deduced molecular parameters such as rotational and centrifugal distortion constants as well as the torsional barrier of the ring methyl group are in agreement with the calculated values.
\end{abstract}

The gas phase structures of toluene and its derivatives have been the subjects of many rotational spectroscopic studies for decades, especially in the microwave and UV frequency regions. Many investigations by UV spectroscopy have been thoroughly carried out for example in Ref. [1-3], giving information on gas phase structures in the ground and excited electronic states of these molecules. In microwave spectroscopy, halogen atoms, ${ }^{[4]}$ aldehyde $^{[5]}$ or alcohol groups ${ }^{[6]}$ are the most common substituents, also providing sufficiently large dipole moment which eases the measurements. Surprisingly, almost no investigations were performed with the methoxy group as substituent. We are interested in these compounds, because from studies in the literature, we found that the internal rotation of methyl groups attached at the benzene ring is difficult to predict. In toluene, only a $V_{6}$ potential is found. ${ }^{[7]}$ In some cases, the $V_{3}$ potential barriers are very low with a large $V_{6}$ contribution..$^{[5]}$ In some other cases, only $V_{3}$ contributions remain. ${ }^{[6]}$ Currently, no study is available to explain systematically this observation.

In the case of mono-substituted methoxy toluenes, which can also be seen as monomethyl substituted anisoles, three relative positions can be ortho, meta, and para, and thus three isomers exist. In the present work, we study the gas phase structure of o-methyl anisole (OMA) with a view towards highly

[a] Dr. Ha Vinh Lam Nguyen

Laboratoire Interuniversitaire des Systèmes Atmosphériques (LISA), CNRS UMR 7583, Université Paris-Est Créteil, Université Paris Diderot, 61 avenue du Général de Gaulle, F-94010 Créteil cedex, France

E-mail: lam.nguyen@lisa.u-pec.fr

[b] Lynn Ferres M. Sc., Prof. Dr. Wolfgang Stahl Institute of Physical Chemistry, RWTH Aachen University, Landoltweg 2, D-52074 Aachen, Germany

[c] Dr. Halima Mouhib

Laboratoire Modélisation et Simulation Multi Echelle (MSME), CNRS UMR 8208, Université Paris-Est, 5 bd. Descartes, F-77454 Marnela-Vallée, France

Supporting information for this article is given via a link at the end of the document. accurate determinations of the barriers to internal rotation. The barrier height of the ring methyl group has only been determined by Alvarez-Valtierra et al. with fluorescence spectroscopy, where the authors found for the electronic ground state a $V_{3}$ potential of $345 \mathrm{~cm}^{-1} \cdot{ }^{[8]}$

The structures of substituted anisoles are also interesting: Anisole itself has a planar heavy atom frame ${ }^{[9]}$ as almost all other related compounds such as phenetole, ${ }^{[10]} p$-fluoroanisole,${ }^{[11]}$ and $p$-anisaldehyde. ${ }^{[12]}$ However, non-planar structures cannot be excluded, because e.g. for p-fluorothioanisole, Lister et al. reported on a second isomer with the methylsulfanyl $\left(\mathrm{CH}_{3}-\mathrm{S}\right)$ group in a nearly perpendicular orientation. ${ }^{[13]}$ Some other molecules containing a phenyl ring also have non planar structures, as in the cases of phenyl formate ${ }^{[14]}$ and $\mathrm{N}$ phenylformamide. ${ }^{[15]}$

Reasonable starting values of molecular parameters such as rotational constants are important for spectral assignment, because reliable predictions of the spectrum considerably simplify the assignment process. Quantum chemistry is a powerful tool for this purpose. The GAUSSIANO9 suite of programs ${ }^{[16]}$ was employed for all calculations in this work. If not stated otherwise, the MP2/6-311++G(d,p) level of theory is used. We choose this level, because it yields quite reasonable results for some other molecules containing aromatic rings like phenetole, ${ }^{[10]} 2,5$ dimethylthiophene, ${ }^{[17]}$ and 2-acetyl-5-methylfuran. ${ }^{[18]}$

Because the rotations of two methyl groups about the $\mathrm{C}_{4}$ $\mathrm{C}_{16}$ and the $\mathrm{O}_{11}-\mathrm{C}_{12}$ bond by varying the dihedral angles $\beta=$ $\angle\left(\mathrm{C}_{5}, \mathrm{C}_{4}, \mathrm{C}_{16}, \mathrm{H}_{19}\right)$ and $\gamma=\angle\left(\mathrm{C}_{3}, \mathrm{O}_{11}, \mathrm{C}_{12}, \mathrm{H}_{14}\right)$, respectively, (for atom numbering see Figure 1) do not create new conformations, the conformational preferences of OMA are solely determined by the rotation of the methoxy group about the $\mathrm{O}_{11}-\mathrm{C}_{3}$ bond by varying the dihedral angle $\alpha=\angle\left(\mathrm{C}_{12}, \mathrm{O}_{11}, \mathrm{C}_{3}, \mathrm{C}_{4}\right)$. A potential curve was calculated where $\alpha$ was varied within a $10^{\circ}$ step width while all other geometry parameters were optimized. The calculated energies were parameterized using a Fourier expansion with the corresponding coefficients given in Table S-1 in the Supporting Information. Using these Fourier coefficients, the potential energy curve was drawn as depicted in Figure 2, showing that there is only one possible conformer for OMA at $\alpha=180^{\circ}$. This conformer has $\mathrm{C}_{\mathrm{s}}$ symmetry, but we note that the minimum of the potential curve is very shallow. Therefore, we recalculate this region within a grid of $1^{\circ}$ and also plotted the result in Figure 2 with an enlarged scale.

Full geometry optimizations and frequency calculations were performed afterwards for the minimum (see Figure 1). The Cartesian coordinates are given in Table S-2 in the Supporting Information. We found one imaginary vibrational mode in the harmonic frequency calculations, which is a bending vibration of the phenyl ring. Stating stable planar ring systems as unstable is a well-known behavior found at the MP2/6-311++G(d,p) level of theory, which has been reported for benzene and arenes. ${ }^{[19]}$ 


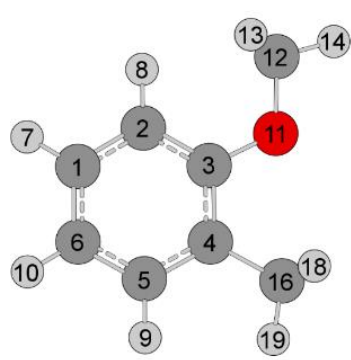

Figure 1. Molecular structure of the only conformer of o-methyl anisole optimized at the MP2/6-311++G(d,p) level of theory. The protons $\mathrm{H}_{15}$ and $\mathrm{H}_{17}$ are located behind $\mathrm{H}_{13}$ and $\mathrm{H}_{18}$, respectively.

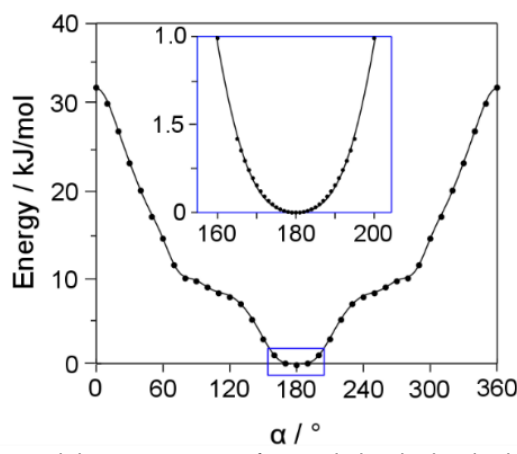

Figure 2. The potential energy curve of $o$-methyl anisole obtained by rotating the methoxy group about the $\mathrm{C}_{3}-\mathrm{O}_{11}$ bond (for atom numbering, see Figure 1) in a grid of $10^{\circ}$. The relative energies with respect to the lowest energy conformation with $E=-385.0454020$ Hartree are given. The minimum region was recalculated in a grid of $1^{\circ}$, as depicted with an enlarged scale.

The only stable conformer of OMA is predicted to possess the rotational constants $A=2.481 \mathrm{GHz}, B=1.559 \mathrm{GHz}$, and $C=$ $0.969 \mathrm{GHz}$ and the dipole moment components $\left|\mu_{a}\right|=0.79 \mathrm{D},\left|\mu_{b}\right|$ $=1.01 \mathrm{D}$, and $\left|\mu_{c}\right|=0.00 \mathrm{D}$. Consequently, no $c$-type transitions are observable in the microwave spectrum and both, $a-$ and $b-$ type transitions, are intense.

In spite of its relatively simple structure, the internal dynamic of OMA is challenging. There are two inequivalent methyl internal rotations. The barrier heights of the ring methyl and the methoxy methyl group were calculated by varying the dihedral angles $\beta$ and $\gamma$, respectively, in a grid of $10^{\circ}$. A rotation of $120^{\circ}$ was sufficient due to the three-fold symmetry of the methyl groups. We found a $V_{3}$ potential of $447.71 \mathrm{~cm}^{-1}$ for the ring methyl and $1118.18 \mathrm{~cm}^{-1}$ for the methoxy methyl group with no significant $V_{6}$ contributions. Simple two-top predictions using the program $X I A M^{[20]}$ indicate that torsional splittings arising from the methoxy methyl group are smaller than $2 \mathrm{kHz}$. Therefore, with the resolution of our spectrometer we expect to observe only splittings arising from the ring methyl group. The potential energy curve of the ring methyl group is shown in Figure S-1; the corresponding coefficients in Table S-3 in the Supporting Information.

The microwave spectrum of the only conformer of OMA was then calculated from the $a b$ initio predictions with the program $X I A M$. As a first step, we neglected the internal rotation effect and used the rigid-rotor mode of XIAM to calculate the theoretical spectra. The dipole moment components suggest a spectrum with intense $a$ - and $b$-type and no $c$-type transitions. We started our assignment with the a-type $R$-branch $J=6 \leftarrow 5$, especially the transitions $6_{06} \leftarrow 5_{05}$ and $6_{16} \leftarrow 5_{15}$, and then the $J=5 \leftarrow 4$ transitions such as $5_{05} \leftarrow 4_{04}$. The assignment of these lines fixed the $B$ and $C$ rotational constants. Afterwards, some $b$-type transitions were also assigned and the $A$ rotational constant was determined.

Characteristic patterns of transitions appeared close to those of the A species, which were assumed to be the $E$ internal rotation symmetry species of the same rotational transitions. In the next step, we took the internal rotation of the ring methyl group into consideration and predicted the spectrum with torsional components using the rotational constants from the rigid rotor fit. The angles $\delta$ between the internal rotor axis and the principal $a$ axis as well as the $V_{3}$ potential were calculated by $a b$ initio. In so doing, the $E$ species frequencies of many transitions were predicted sufficiently accurate, especially for a-type transitions, leading to their straightforward assignment. Finally, we could assign and fit 30 torsional lines in the $10-14 \mathrm{GHz}$ broadband spectrum (shown in Figure 3). Using the prediction refined from this fit, high resolution measurements were performed in the frequency range $2-26.5 \mathrm{GHz}$, leading to an expanded assignment of $125 \mathrm{~A}$ and $119 \mathrm{E}$ lines and a standard deviation of $2.9 \mathrm{kHz}$. Figure 4 shows typical transitions of OMA measured at high resolution. The fitted molecular parameters are summarized in Table 1. All fitted transitions are listed in Table S-4 in the Supporting Information.

The three linear combinations of the rotational constants $B J$ $=1 / 2(B+C), B_{K}=A-1 / 2(B+C), B_{-}=1 / 2(B-C)$ and the quartic centrifugal distortion constants were determined with very high accuracy by fitting 244 torsional transitions in a one-rotor fit. The $V_{3}$ potential, the angle $\delta$ between the internal rotor axis and the principal $a$ axis, and two higher order parameters $D_{\text {pizJ }}$ and $D_{\text {piz- }}$ were also obtained. The internal rotation constant $F_{0}$ of the methyl top could not be fitted because the present data set contains only information on the torsional ground state with relatively low $J$ and $K$ values, and because the torsional barrier is rather high $\left(459 \mathrm{~cm}^{-1}\right)$.

Table 1. Molecular parameters of $o$-methyl anisole obtained by the program XIAM.

\begin{tabular}{llrrr}
\hline Parameter $^{\mathrm{a}}$ & Unit & \multicolumn{1}{l}{ Obs. } & \multicolumn{1}{l}{ Calc. $^{\text {b }}$} & Obs. - Calc. \\
\hline$A$ & $\mathrm{MHz}$ & $2489.335(87)$ & 2481.1078 & 8.23 \\
$B$ & $\mathrm{MHz}$ & $1557.68(17)$ & 1558.5760 & -0.90 \\
$C$ & $\mathrm{MHz}$ & $970.516(37)$ & 968.9111 & 1.60 \\
$D_{J}$ & $\mathrm{kHz}$ & $0.03845(63)$ & 0.03655 & 0.0019 \\
$D_{J k}$ & $\mathrm{kHz}$ & $0.1583(23)$ & 0.17364 & 0.0153 \\
$D_{K}$ & $\mathrm{kHz}$ & $0.1091(28)$ & 0.03958 & 0.0695 \\
$d_{1}$ & $\mathrm{kHz}$ & $-0.01792(33)$ & -0.01802 & 0.0001 \\
$d_{2}$ & $\mathrm{kHz}$ & $-0.006296(92)$ & -0.00668 & 0.0004 \\
$V_{3}$ & $\mathrm{~cm}-1$ & $444.05(41)$ & 459.25 & -15.20 \\
$F_{0}$ & $\mathrm{GHz}$ & $158.0^{\mathrm{c}}$ & 157.9223 & 0.1 \\
$D p i_{2 J}$ & $\mathrm{kHz}$ & $60.0(7.0)$ & & \\
$D p i_{2-}$ & $\mathrm{kHz}$ & $43.6(6.4)$ & & \\
$\angle(i, a)$ & $\circ$ & $70.996(95)$ & 68.8415 & 2.154 \\
$\angle(i, b)$ & $\circ$ & $19.004(95)$ & 21.1586 & -2.155 \\
$\angle(i, c)$ & $\circ$ & $90.0^{\mathrm{d}}$ & 89.970 & 0.030 \\
$N_{A} / N_{E}{ }^{\mathrm{e}}$ & $\circ$ & $125 / 119$ & & \\
$\sigma^{f}$ & $\mathrm{kHz}$ & 2.9 & & \\
\hline
\end{tabular}

a All parameters refer to the principal axis system. Watson's S reduction and Ir representation were used. ${ }^{b}$ Centrifugal distortion constants obtained from anharmonic frequency calculations at the B3LYP/6-311++G(d,p) level of theory, all other values from geometry optimizations at the MP2/6$311++\mathrm{G}(\mathrm{d}, \mathrm{p})$ level. c Corresponds to $I_{\alpha}=3.2 \mathrm{u} \AA^{2}$, a value often found for methyl groups. ${ }^{d}$ Fixed due to $C_{s}$ symmetry. ${ }^{e}$ Number of $A$ and $E$ species lines. ${ }^{\dagger}$ Standard deviation of the fit. 


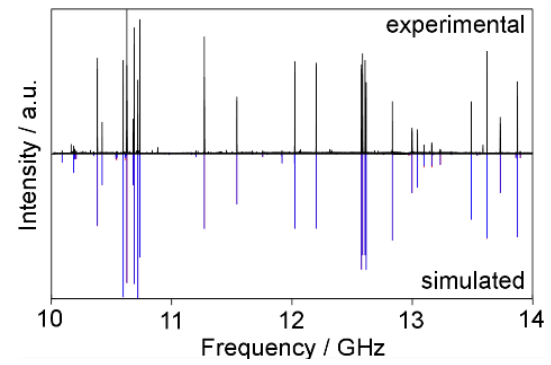

Figure 3. A broadband scan of o-methyl anisole from 10 to $14 \mathrm{GHz}$. The experimental spectrum is the upper trace. The lower trace indicates the theoretical spectrum predicted using the molecular parameters deduced from a one-top fit using the program XIAM, showing that (i) a simple one-top model can reasonably reproduce the microwave spectrum of $o$-methyl anisole, and (ii) only some weak lines remained unassigned in the spectrum.

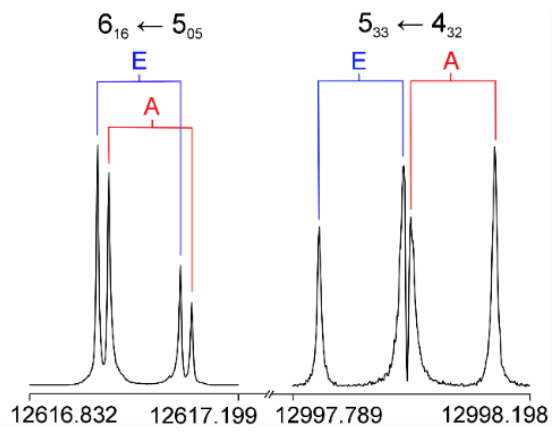

Figure 4. Typical $b$-type and a-type transitions (in $\mathrm{MHz}$ ) of o-methyl anisole measured at high resolution. The measurement accuracy is $2 \mathrm{kHz}$, the typical experimental line width $20 \mathrm{kHz}$. The doublets marked by brackets are due to the Doppler effect. The torsional A-E splittings are also indicated. For both spectra, 50 free-induction decays were co-added.

The experimentally deduced rotational constants are compared with those from quantum chemical calculations (see Table 1). The experimental and calculated $B$ and $C$ rotational constants agree well and the deviation in the $A$ rotational constant is very small (about $8 \mathrm{MHz}$ ). The calculated $V_{3}$ potential of 459 $\mathrm{cm}^{-1}$ is also quite close to the experimental value of $444 \mathrm{~cm}^{-1}$. The centrifugal distortion constants obtained by anharmonic frequency calculations at the B3LYP/6-311++G(d,p) level of theory are in good agreement with the experimental values. Because the benzene frame of OMA is quite rigid, all centrifugal distortion constants are small. Calculations with the MP2 and B3LYP methods in combination with various basis sets were performed in addition, which confirm that the MP2/6-311++G(d,p) level is sufficiently suitable for OMA, as can be seen in Table S-5 in the Supporting Information. It is noteworthy that the structures obtained from calculations at all levels of theory are consistent, which is not obvious if compared to the case of e.g. diethyl ketone. ${ }^{[21]}$

In 2006 Alvarez-Valtierra et al. determined the rotational constants $A=2.4900$ (1) $\mathrm{GHz}, B=1.5589(1) \mathrm{GHz}$, and $C=$ $0.9707(1) \mathrm{GHz}$ as well as a $V_{3}$ potential of $345 \mathrm{~cm}^{-1}$ for OMA by fluorescence spectroscopy. ${ }^{[8]}$ The rotational constants are not as accurate as in the present work, but they are in agreement with those deduced from our fit. On the other hand, the rotational barrier determined by fluorescence spectroscopy is $99 \mathrm{~cm}^{-1}$ lower than our value, which is a significant difference. We believe that the barrier height given by microwave spectroscopy is more reliable, since this parameter was only indirectly determined in fluorescence spectroscopy.

It is interesting to compare the $V_{3}$ potential of the ring methyl group in OMA with those of other toluene derivatives, where the substituents are also in the ortho position with respect to each other (see Table 2). In the case of acetates, we found that both the steric and electronic effects might affect the methyl barrier height, where the electronic effect has a larger influence than the steric effect. ${ }^{[22]}$ In the case of ortho substituted toluene, the influence of the substituent on the barrier height seems to be predominantly of a steric nature. Small or slim substituents such as fluorine atoms (in o-fluorotoluene $e^{[23]}$ or 2,4-difluorotoluene ${ }^{[4]}$ ) or cyano groups (o-tolunitrile ${ }^{[24]}$ ) hinder the internal rotation much less than voluminous chlorine atoms (o-chlorotoluene ${ }^{[25]}$ ), hydroxyl groups (o-cresol $\left.{ }^{[6]}\right)$, methyl groups (o-xylene ${ }^{[26]}$ or $3,4-$ dimethylbenzaldehyde ${ }^{[27]}$ ), methoxy groups (OMA), or aldehyde groups (2,5-dimethylbenzaldehyde $\left.{ }^{[27]}\right)$. In molecules where synand anti-conformers co-exist in the spectrum, the anti-conformer has lower internal rotation barrier, because the ortho substituent is much closer to the methyl group, and the steric hindering increases, while the electronic contribution should be similar for both conformers. An electronic effect is, however, not excluded, as can be seen from the different $V_{3}$ potential of $o$-fluorotoluene ${ }^{[23]}$ and 2,4-difluorotoluene $e^{[4]}$ or $o$-xylene $e^{[26]}$ and 3,4-dimethylbenzaldehyde. ${ }^{[27]}$ Nevertheless, these differences are less significant.

The planar moment of inertia $P_{c c}=-2\left(I_{c}-I_{a}-I_{b}\right)=13.396$ $u \AA^{2}$ of OMA confirms that the heavy atom skeleton is planar with two pairs of hydrogen atoms out of plane. These values are very similar as that found in other planar molecules containing two methyl groups such as the trans and cis conformers of 2-acetyl5-methylfuran $\left(P_{c c}=12.938 \text { and } 13.160 \mathrm{uA}^{2} \text {, respectively }\right)^{[18]}$ and the syn and anti conformers of 3,4-dimethylbenzaldehyde ( $P_{c c}=$ 12.904 and $12.938 u \AA^{2}$, respectively). ${ }^{[27]}$

After the spectral assignment, only very few weak lines remained unassigned in the broadband scan, as shown in Figure 3. We thus concluded that water complexes or dimers were not present under our measurement conditions.

Table 2. Torsional barriers of ortho substituted toluene derivatives.

\begin{tabular}{lcr}
\hline & $V_{3} / \mathrm{cm}^{-1}$ & Ref. \\
\hline o-tolunitrile & $187.699(3)$ & {$[24]$} \\
o-fluorotoluene & $227.28(2)$ & {$[23]$} \\
2,4-difluorotoluene & $203.91(24)$ & {$[4]$} \\
anti-o-cresol & $371.05(41)$ & {$[6]$} \\
syn-o-cresol & $669.10(51)$ & {$[6]$} \\
o-methyl anisole & $444.05(41)$ & this work \\
anti-3,4-dimethylbenzaldehyde & $454.1(14), 480.6(44)$ & {$[27]$} \\
syn-3,4-dimethylbenzaldehyde & $508.1(11), 550.7(88)$ & {$[27]$} \\
${ }^{37} \mathrm{Cl}-$-chlorotoluene & $507.2(83)$ & {$[25]$} \\
${ }^{35} \mathrm{Cl}-$-chlorotoluene & $513.8(27)$ & {$[25]$} \\
o-xylene & $518.3(32)$ & {$[26]$} \\
syn-2,5-dimethylbenzaldehyde & $566(16)$ & {$[27]$} \\
\hline
\end{tabular}




\section{Experimental section}

A broadband scan from 10 to $14 \mathrm{GHz}$ was recorded, where overlapping spectra with a step width of $250 \mathrm{kHz}$ and 50 co-added free-induction decays were automatically taken using a molecular jet Fourier-transform microwave spectrometer operating in the frequency range from 2 to $26.5 \mathrm{GHz} .{ }^{[28]}$ OMA was purchased from $\mathrm{TCl}$ Europe, Eschborn, Germany, with a stated purity of $98 \%$. Without any further purification, the substance was placed on a 5 $\mathrm{cm}$ piece of a pipe cleaner inside a stainless steel tube mounted upstream the nozzle because of its low vapor pressure. Under helium stream at a backing pressure of $200 \mathrm{kPa}$, the OMA-He mixture was expanded into the cavity. All lines in the broadband scan (Figure 3) were subsequently remeasured at higher resolution with an intrinsic experimental line width of $2 \mathrm{kHz}$ for isolated lines, ${ }^{[29]}$ indicating completely resolved torsional splittings due to internal rotation of the ring methyl group (Figure 4). The average value of the line widths is about $20 \mathrm{kHz}$, corresponding to a measurement accuracy of $2 \mathrm{kHz}$. The line broadening was probably due to unresolved proton hyperfine splittings or unresolved splittings arising from the internal rotation of the methoxy methyl group. All rotational signals appeared in addition to the torsional splittings as doublets due to the Doppler effect. The molecular transition frequency is the center frequency of these doublets.

\section{Acknowledgments}

We thank the Land Nordrhein-Westfalen for funds. Simulations were performed with computing resources granted by RWTH Aachen University under project <thes0126>. H.M. thanks the Excellence Initiative grants of the German federal and state governments (StUpPD_209_15).

Keywords: structure determination - microwave spectroscopy • internal rotation $\cdot$ rotational spectroscopy

[1] I. Kalkman, C. Vu, M. Schmitt, W. L. Meerts, Phys. Chem. Chem. Phys. 2009, 11, 4311-4318.

[2] M. Böhm, C. Ratzer, M. Schmitt, J. Mol. Struct. 2006, 800, 55-61.

[3] R. M. Helm, H.-P. Vogel, H. J. Neusser, Chem. Phys. Lett. 1997, 270, 285-292.

[4] S. Maiti, A. I. Jaman, R. N. Nandi, J. Mol. Spectrosc. 1996, 177, $29-33$.

[5] A. J. Shirar, D. S. Wilcox, K. M. Hotopp, G. L. Storck, I. Kleiner, B. C. Dian, J. Phys. Chem. A 2010, 114, 12187-12194.

[6] A. Welzel, A. Hellweg, I. Merke and W. Stahl, J. Mol. Spectrosc. 2002 $215,58-65$.

[7] V. V. Ilyushin, Z. Kisiel, L. Pszczólkowski, H. Mäder, J. T. Hougen, J. Mol. Spectrosc. 2010, 259, 26-38.

[8] L. Alvarez-Valtierra, J. T. Yi, D. W. Pratt, J. Phys. Chem. B 2006, 110, 19914-19922

[9] M. Onda, A. Toda, S. Mori, I. Yamaguchi, J. Mol. Struct. 1986, 144, 4751.

[10] L. Ferres, W. Stahl, H. V. L. Nguyen, Mol. Phys. 2016, 114, 2788-2793.

[11] D. G. Lister, J. Mol. Struct. 1980, 68, 33-40.

[12] R. K. Bohn, M. S. Farag, C. M. Ott, J. Radhakrishnan, S. A. Sorenson, N. S. True, J. Mol. Struct. 1992, 268, 107-121.

[13] D. G. Lister, P. Palmieri, C. Zauli, J. Mol. Struct. 1976, 35, 299-302.

[14] L. Ferres, H. Mouhib, W. Stahl, M. Schwell, H. V. L. Nguyen, J. Mol. Spectrosc. 2017, doi: 10.1016/j.jms.2017.04.017.

[15] J.-R. A. Moreno, D. Petitprez, T. R. Huet, Chem. Phys. Lett. 2006, 419, 411-416.
[16] M. J. Frisch, G. W. Trucks, H. B. Schlegel, G. E. Scuseria, M. A. Robb J. R. Cheeseman, G. Scalmani, V. Barone, B. Mennucci, G. A. Petersson, H. Nakatsuji, M. Caricato, X. Li, H. P. Hratchian, A. F. Izmaylov, J. Bloino, G. Zheng, J. L. Sonnenberg, M. Hada, M. Ehara, K. Toyota, R. Fukuda, J. Hasegawa, M. Ishida, T. Nakajima, Y. Honda, O. Kitao, H. Nakai, T. Vreven, J. A., Jr. Montgomery, J. E. Peralta, F. Ogliaro, M. Bearpark, J. J. Heyd, E. Brothers, K. N. Kudin, V. N. Staroverov, R. Kobayashi, J. Normand, K. Raghavachari, A. Rendell, J. C. Burant, S. S. lyengar, J. Tomasi, M. Cossi, N. Rega, J. M. Millam, M. Klene, J. E. Knox, J. B. Cross, V. Bakken, C. Adamo, J. Jaramillo, R. Gomperts, R. E. Stratmann, O. Yazyev, A. J. Austin, R. Cammi, C. Pomelli, J. W. Ochterski, R. L. Martin, K. Morokuma, V. G. Zakrzewski, G. A. Voth, P. Salvador, J. J. Dannenberg, S. Dapprich, A. D. Daniels, O. Farkas, J. B. Foresman, J. V. Ortiz, J. Cioslowski, D. J. Fox, Gaussian 09, Revision A.02, Gaussian, Inc., Wallingford CT, 2009.

[17] V. Van, W. Stahl, H.V.L. Nguyen, Phys. Chem. Chem. Phys. 2015, 17, 32059-32578.

[18] V. Van, W. Stahl, H.V.L. Nguyen, ChemPhysChem 2016, 17, 32233228.

[19] D. Moran, A. C. Simmonett, F. E. Leach, W. D. Allen, P. v. R. Schleyer, H. F. Schaefer, J. Am. Chem. Soc. 2006, 128, 9342-9343.

[20] H. Hartwig, H. Dreizler, Z. Naturforsch. 1996, 51a, 923-932.

[21] H.V.L. Nguyen, W. Stahl, ChemPhysChem 2011, 12, 1900-1905

[22] A. Jabri, V. Van, H. V. L. Nguyen, W. Stahl, I. Kleiner, ChemPhysChem 2016, 17, 2660-2665.

[23] S. Jacobsen, U. Andresen, H. Mäder, Struct. Chem. 2003, 14, 217-225.

[24] N. Hansen, H. Mäder, T. Bruhn, Mol. Phys. 1999, 97, 587-595.

[25] K. P. R. Nair, J. Demaison, G. Wlodarczak, I. Merke, J. Mol. Spectrosc. 2006, 237, 137-142.

[26] H. D. Rudolph, K. Walzer, I. Krutzik, J. Mol. Spectrosc. 1973, 47, 314339.

[27] M. Tudorie, I. Kleiner, M. Jahn, J.-U. Grabow, M. Goubet, O. Pirali, J. Phys. Chem. A 2013, 117, 13636-13647.

[28] J.-U. Grabow, W. Stahl, H. Dreizler, Rev. Sci. Instrum. 1996, 67, 40724084.

[29] J.-U. Grabow, W. Stahl, Z. Naturforsch. 1990, 45a, 1043-1044 
Entry for the Table of Contents (Please choose one layout)

Layout 1:

\section{COMMUNICATION}

Rotating on the ring - the methyl torsional barrier

Torsional splittings due to the internal rotation of the ring methyl group of 0 methyl anisole were analyzed by microwave spectroscopy. The barrier to internal rotation was accurately determined. Results from quantum chemistry are in agreement with the experiments.

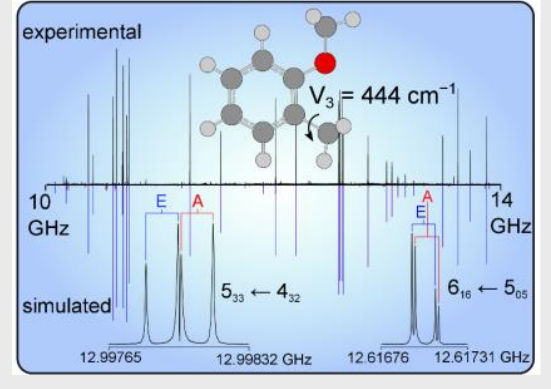

Lynn Ferres, Halima Mouhib, Wolfgang Stahl, Ha Vinh Lam Nguyen*

Page No. - Page No.

Methyl Internal Rotation in the Microwave Spectrum of o-Methyl Anisole 